\title{
An Approach to Improve the Performance of Mobile Computation Technology Using Data Offloading
}

\begin{tabular}{|c|c|}
\hline $\begin{array}{l}\text { Md. Ashraful Islam }{ }^{1} \\
\text { Md. Mostofa Kamal Tareq } \\
\text { Ishrat Khan Mohona }^{3} \\
\text { Ifthekhar Ahammad }^{4} \\
\text { Mohammad Shamim Kaiser }^{5}\end{array}$ & \\
\hline $\begin{array}{l}\text { 1,4Leading University, Sylhet, Bangladesh } \\
\text { 2Incepta Vaccine Ltd, Bangladesh } \\
\text { 3University of Dhaka, Bangladesh } \\
5 \text { Jahangirnagar University, Bangladesh }\end{array}$ & \\
\hline
\end{tabular}

\begin{abstract}
Modern mobile phones have a powerful processing unit that can perform multiple operations simultaneously. But the main constrain is the processing power and energy required to drive it. Cloud computing has come as a blessing for the mobile computing as it possesses a vast resource, high storage capacity and high processing power. In Mobile Cloud Computing (MCC), computation is offloaded to the cloud, cloud processes the data and generates information; and sends it back to the mobile device. Probability of offloading depends on line bandwidth, line length, size of data, processing speed of mobile, processing speed of cloud, storage and energy capacity of mobile etc. This paper presents a model for efficient data offloading decision, depending on the total execution time of a task when the data computation happens in the mobile and when it is offloaded to the cloud. An algorithm has been proposed here on data offloading decision. As thus an equation has been proposed to observe the probability of offloading process.
\end{abstract}

Keywords: Cloud computing, Offloading, Data computation, Bandwidth, Processing speed.

\section{Contents}

1. Introduction .

2. Offloading .....

3. Methodology

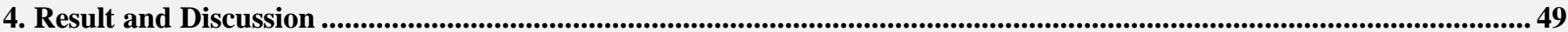

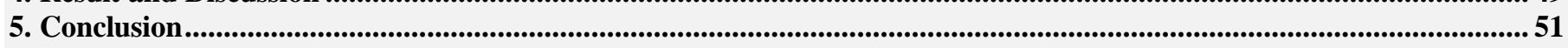

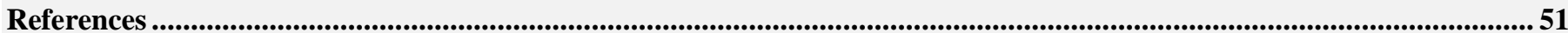

Citation | Md. Ashraful Islam; Md. Mostofa Kamal Tareq; Ishrat Khan Mohona; Ifthekhar Ahammad; Mohammad Shamim Kaiser (2017). An Approach to Improve the Performance of Mobile Computation Technology Using Data Offloading. International Journal of Modern Research in Electrical and Electronic Engineering, 1(1): 47-52.

DOI:

Licensed: 10.20448/journal.526.2017.11.47.52 Crossref

Contribution/Acknowledgement: Funding: This work is licensed under a Creative Commons Attribution 3.0 License $(\mathrm{cc}) \mathrm{EY}$

Competing Interests: All authors contributed to the conception and design of the study.

Transparency: This study received no specific financial support.

Transparency:

The authors declare that they have no conflict of interests.

History:

Ethical:

Publisher:

The authors confirm that the manuscript is an honest, accurate, and transparent account of the study was reported; that no vital features of the study have been omitted; and that any discrepancies from the study as planned have been explained. Received: 7 March 2017/ Revised: 28 March 2017/ Accepted: 31 March 2017/ Published: 5 April 2017 This study follows all ethical practices during writing. Asian Online Journal Publishing Group 


\section{Introduction}

Cloud computing, in a whole, refers the sharing of application, platform and infrastructure. Remote servers are connected to make a broad network to share software, hardware and storage [1]. Users from anywhere having the access to this network can use this resource any time. This facilitates every user with cost effectiveness (CE), mobility, high computation power etc. In cloud based computation, end users have different devices e.g. smartphone, computer, tablet, video game console, smart TV, printer, security camera etc. but everybody can utilize the same services like data processing and storage, arranged in a central way. End users offload data to the cloud where data is analyzed according to the user requirement and resultant data is sent back to the sender. As the amount of computational data increases, the probability of using cloud computation increases. This probability also depends on bandwidth of communication line, end user's processing speed and cloud processing speed. With the advancement of mobile applications, large amount of data processing in a short time has become a big concern. Large amount of computation demands availability of complex hardware, large storage, reliable software and huge power sources which make a mobile device costly and inconvenient. So, hardware free computation is the most important factor that makes cloud computing unique [2].

Data offloading is an important and basic factor of cloud computing. A lot of research has already been done in recent past. So far Offloading policy has been studied extensively. In Valeria Cardellini's research [3] consideration has been made with three tier architecture, a system model has been proposed to capture the user's interaction with the external servers and investigating the effects of computation offloading. A data offloading algorithm has been proposed in Amin \& Mahtab's work [4] to assign offloaded mobile stations to access points, based on mapping the problem to find a perfect matching algorithm. In another research [5] a technique is introduced to automatically generate accurate and efficient method-wise performance predictors for mobile applications to enhance the performance of offloading. In Xing \& Yuan's study [6] collaborative task execution between mobile devices and cloud has been considered and a scheduling algorithm has also been provided to solve the optimization problem. In both K. Kumar's [7] and Feng's [8] research, the offloading policy has been investigated, whether the data/task is to be offloaded to cloud or to be processed in mobile phone in terms of computation execution time and energy consumption.

In this paper, we analyze the data computation time in two scenarios. In the first scenario, we consider data to be processed in mobile and in the second scenario we consider data to be off-loaded to the cloud, processed in the cloud and on-loaded (processed data) to the mobile. We observed that all the previous researches barely considered the propagation delay/ path delay while off-loading. In this paper, we consider the path delay in cloud computing process. An algorithm has been proposed to determine whether the data is needed to be offloaded, in terms of data execution time. Offloading probability hugely depends on the size of total data to be computed and offered bandwidth. At the later part of this paper an equation has also been proposed to observe how the probability changes with respect to data size and bandwidth.

\section{Offloading}

With the progressive advancement of mobile technology, the users of computer are expanding their workstations from desktops to a greater range of mobile devices- like mobile phones, surveillance, environmental sensing, etc. But here, mobile devices are battery powered, have less memory, and slow processor. Low bandwidth in wireless communication is one of the main challenges for data offloading. These limitations have been creating the gap between the demand of complex programs and the availability of resources [9].

By offloading our computation (Figure 1) these limitations could be overcome. Offloading increases the capabilities of a mobile system by shifting the work i.e. computation, to a more resourceful cloud or server [7].

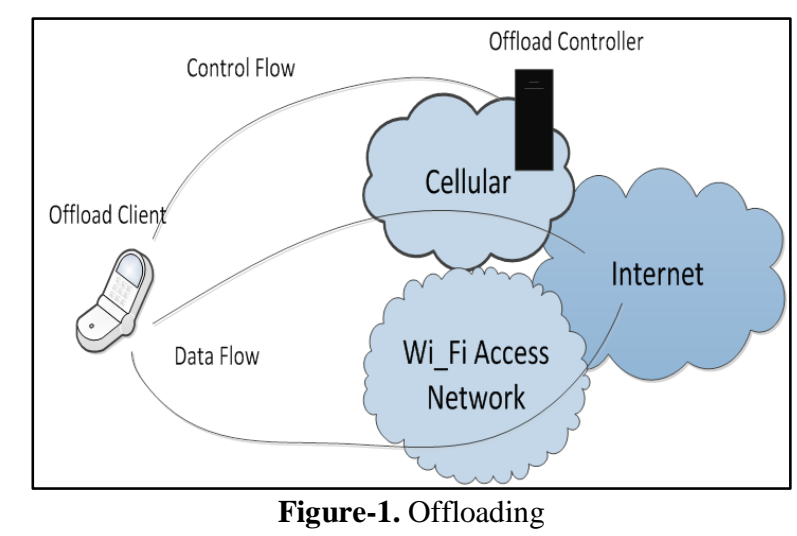

Since offloading shifts computation to a more resourceful server, decision should have been made: of when and what computation, someone should offload. These decisions have to be taken considering performance of the mobile device and energy efficiency.

\section{Methodology}

To analyze the scenario a consideration has been made where a mobile phone is connected to a cloud server with some routers or access points (black dots). Bandwidth and distance of paths connecting the routers are denoted by B and $\mathrm{D}$ respectively.

Assuming,

Total Data Computation, $W=50 \mathrm{MB}$

Processing speed of the mobile system, $\mathrm{S}_{\mathrm{m}}=50 \mathrm{MB} / \mathrm{s}$

Sending data, $d_{s}=20 \mathrm{MB}$ 
Processing speed in cloud, $\mathrm{S}_{\mathrm{c}}=200 \mathrm{MB} / \mathrm{s}$

Receiving data, $\mathrm{d}_{\mathrm{r}}=5 \mathrm{MB}$

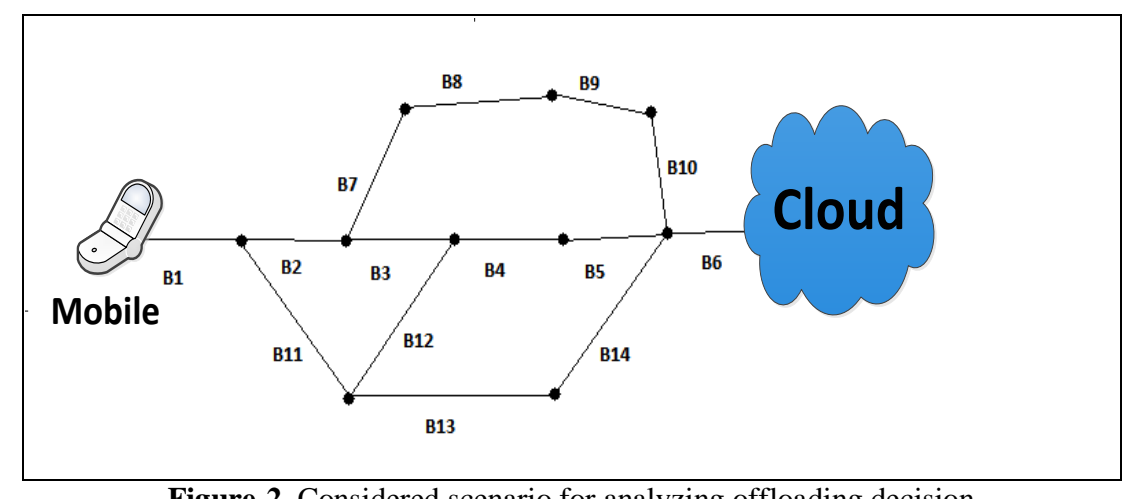

Figure-2. Considered scenario for analyzing offloading decision

The paths are,

1. $\mathrm{D}_{1} \mathrm{D}_{2} \mathrm{D}_{3} \mathrm{D}_{4} \mathrm{D}_{5} \mathrm{D}_{6}$

2. $\mathrm{D}_{1} \mathrm{D}_{11} \mathrm{D}_{13} \mathrm{D}_{14} \mathrm{D}_{6}$

3. $\mathrm{D}_{1} \mathrm{D}_{11} \mathrm{D}_{12} \mathrm{D}_{4} \mathrm{D}_{5} \mathrm{D}_{6}$

4. $\mathrm{D}_{1} \mathrm{D}_{2} \mathrm{D}_{7} \mathrm{D}_{8} \mathrm{D}_{9} \mathrm{D}_{10} \mathrm{D}_{6}$

And,

$\mathrm{B}_{1}=\mathrm{B}_{5}=\mathrm{B}_{10}=\mathrm{B}_{14}=500 \mathrm{MHz}$

$\mathrm{B}_{2}=\mathrm{B}_{4}=\mathrm{B}_{9}=\mathrm{B}_{13}=400 \mathrm{MHz}$

$\mathrm{B}_{3}=\mathrm{B}_{6}=\mathrm{B}_{8}=\mathrm{B}_{11}=\mathrm{B}_{12}=450 \mathrm{MHz}$ and

$\mathrm{D}_{1}=\mathrm{D}_{6}=\mathrm{D}_{11}=10 \mathrm{~km}$

$\mathrm{D}_{2}=\mathrm{D}_{10}=\mathrm{D}_{12}=\mathrm{D}_{14}=8 \mathrm{~km}$

$\mathrm{D}_{3}=\mathrm{D}_{5}=\mathrm{D}_{13}=15 \mathrm{~km}$

$\mathrm{D}_{4}=\mathrm{D}_{8}=\mathrm{D}_{9}=12 \mathrm{~km}$

Here, Smallest Bandwidth is $400 \mathrm{MHz}$

\section{Result and Discussion}

A. Equations and Algorithm

To get the data computation time in a mobile device, it is only needed to compute the processing time in that device.

Data computation time in mobile device [7]; [10].

$T_{m}=\frac{\text { Computation data }}{\text { Processing speed of mobile }}=\frac{W}{S_{m}}$

And to get data computation time while offloading, we have to consider queue delay, path delay and processing time in cloud [7]; [10].

Here,

Sending queue delay $=\frac{d_{s}}{B_{3}}$

Receiving queue delay $=\frac{d_{r}}{B_{3}}$

Path delay $=\frac{2 D}{c}$, where $c$ is the velocity of light and $\mathrm{D}$ is the distance of respective paths (For $1^{\text {st }}$ path, $\mathrm{D}=\mathrm{D}_{1+} \mathrm{D}_{2+}$ $\left.\mathrm{D}_{3+} \mathrm{D}_{4+} \mathrm{D}_{5}+\mathrm{D}_{6}\right)$.

Processing time in cloud $=\frac{W}{S_{c}}$

So, Data computation time while offloading,

$$
T_{0}=T_{\text {offload }}+\text { Processing time in cloud }+T_{\text {onload }}
$$

(Sending queue delay + Sending path delay $)+$ (Receiving queue delay +

$=\left(\frac{d_{s}}{B_{3}}+\frac{D}{c}\right)+\frac{W}{S_{c}}+\left(\frac{d_{r}}{B_{3}}+\frac{D}{c}\right)$ Receiving path delay)

According to equation (1), computation time in mobile of our considered scenario is 1 second.

To compute in cloud, we have four different possible paths. According to equation (2):

For $1^{\text {st }}$ path, $T_{0}=0.3129666667 \mathrm{sec}$

For $2^{\text {nd }}$ path, $T_{0}=0.3128533333 \mathrm{sec}$

For $3^{\text {rd }}$ path, $T_{0}=0.3129333333 \mathrm{sec}$

For $4^{\text {th }}$ path, $T_{0}=0.3129666667 \mathrm{sec}$

It is obvious that $2^{\text {nd }}$ path has the shortest delay. This calculation clearly shows that the overall data computation time in mobile $(1 \mathrm{sec})$ is slower than the $2^{\text {nd }}$ offloading path $(0.3128533333 \mathrm{sec})$. On the considered scenario, it has been seen that total execution time while offloading $\&$ on-loading is much less than the computation time in mobile. So, an algorithm has been proposed for offloading decision (Figure 3 ). 


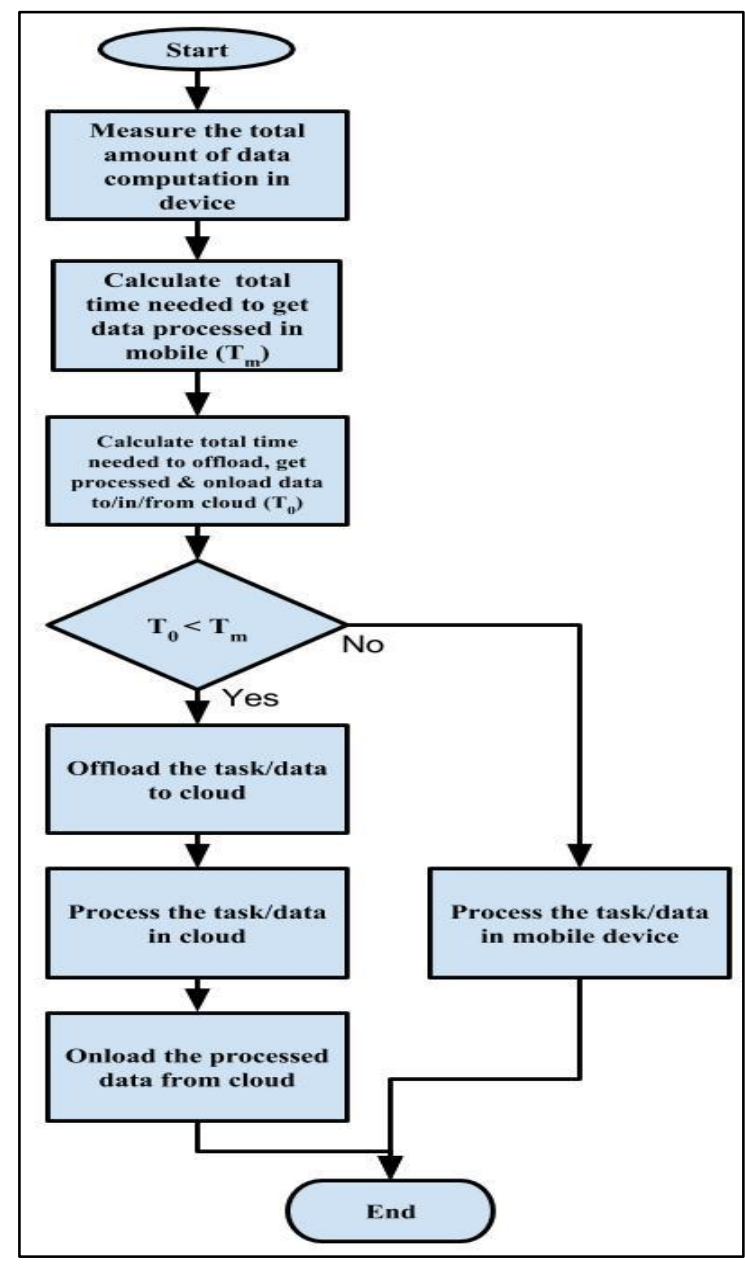

Figure-3. Algorithm for offloading decision

\section{B. Graphical Representation:}

\section{Mobile computation:}

Consider Data computation $W=50 M B$ and using equation (1), we get the plot in Figure 4 .

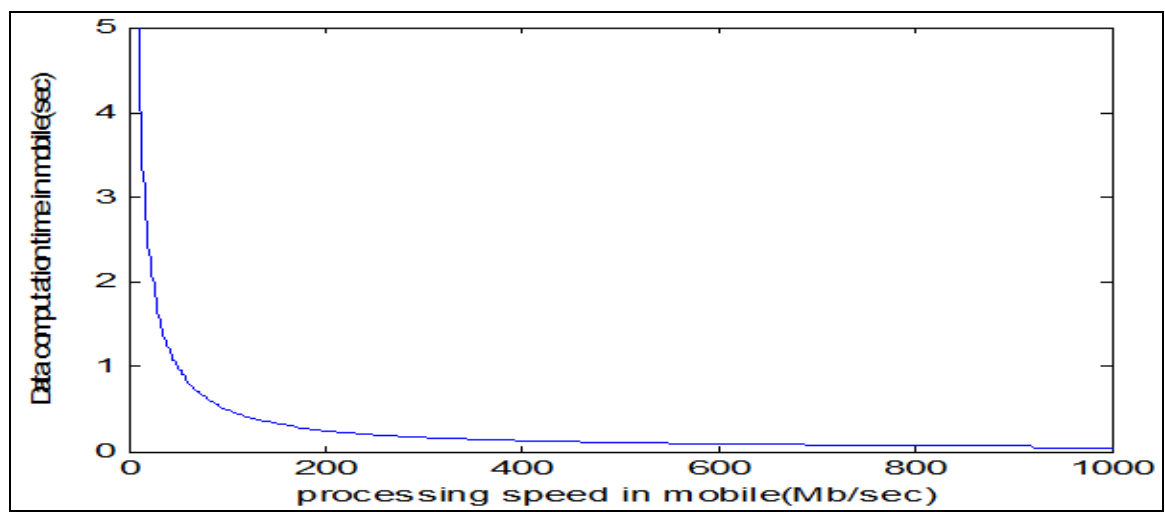

Figure-4. Plot of computation time in mobile (sec) vs. processing speed in mobile (MB/sec)

Figure-4 shows that if the processing speed in mobile device increases, the data processing time decreases.

\section{Cloud computation:}

Considering $d_{s}=20 \mathrm{MB} ; \mathrm{W}=50 \mathrm{MB} ; \mathrm{D}=53 \mathrm{KM} ; \mathrm{S}_{\mathrm{c}}=200 \mathrm{MB} / \mathrm{s} ; \mathrm{d}_{\mathrm{r}}=5 \mathrm{MB}$. From equation (2) we get the plot of Figure 5, which shows data computation time with respect to line bandwidth.

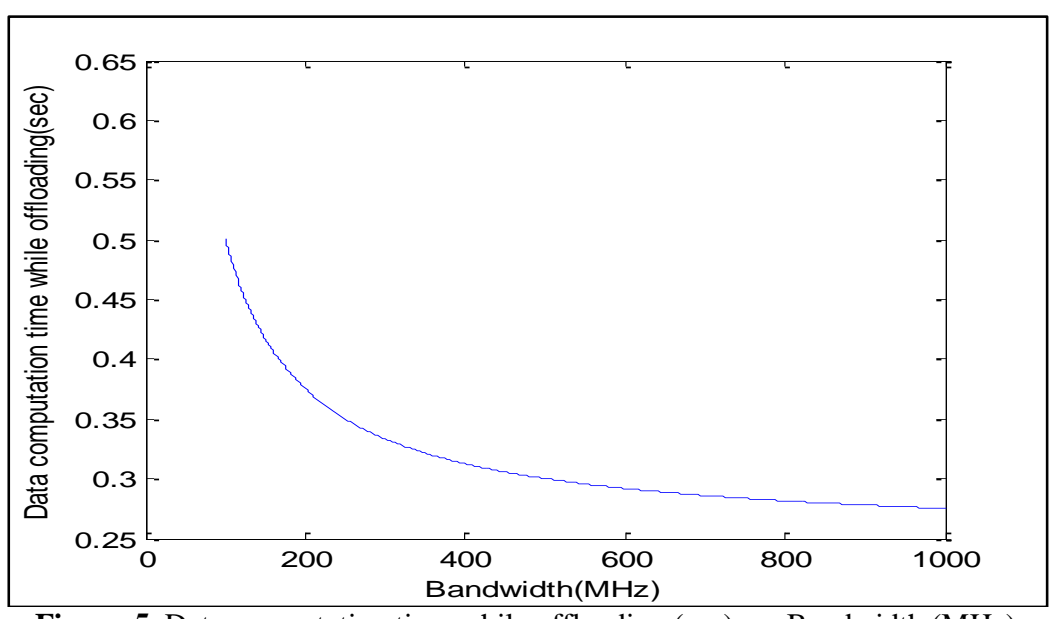

Figure-5. Data computation time while offloading (sec) vs. Bandwidth (MHz) 
Figure-5 shows that the higher the line bandwidth is, the lower time it takes to compute the data. This figure clearly shows why the line bandwidth is so important in cloud computing.

Considering $d_{s}=20 \mathrm{MB} ; B=400 \mathrm{MHz} ; \mathrm{W}=50 \mathrm{MB} ; \mathrm{D}=53 \mathrm{KM} ; \mathrm{d}_{\mathrm{r}}=5 \mathrm{MB}$. From equation (2) we get the plot of Figure 6, which shows the relation of data computation time with respect to processing speed in cloud.

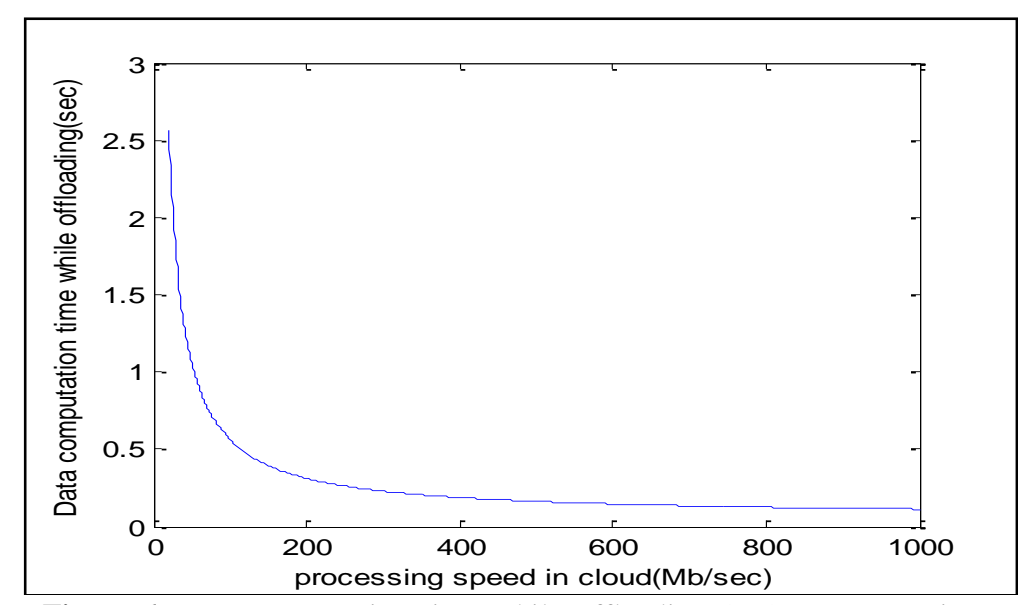

Figure-6. Data computation time while offloading (sec) vs. processing speed in cloud $(\mathrm{MB} / \mathrm{sec})$

We can understand from Figure-6 that if the processing speed in cloud/server is high, it will take less time to compute the data.

\section{Probability of offloading}

Offloading probability (OP) depends on two parameters- "Data computation" and "Offered bandwidth". Consider maximum data computation is $100 \mathrm{~GB}$ and maximum offered bandwidth is $10^{6} \mathrm{~Hz}$ [11]. For this scenario, we have chosen not to offload until $10 \%$ of maximum data is to be computed or $10 \%$ of maximum BW is offered. Probability follows the equation: $y=e^{x-0.1}-1$.

Where, $y=$ probability to offload.

$x=$ normalized computation or bandwidth.

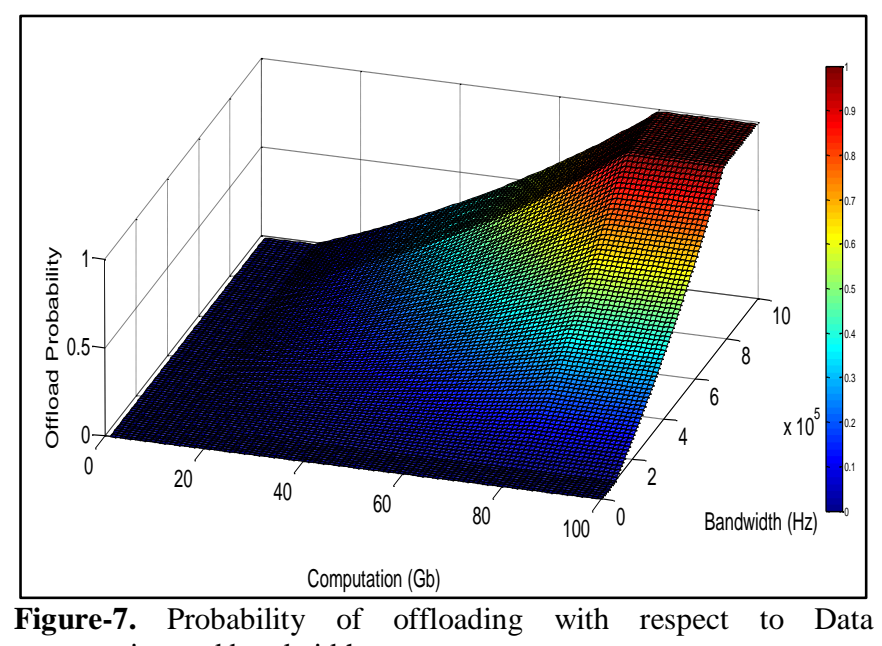

computation and bandwidth

OP increases exponentially with the increase of data computation and offered bandwidth. This proposed equation shows that if the amount of data computation is large and offered bandwidth is high, then probability of offloading is also high. This 3D plot (Figure-7) shows every possible scenario as the product of two probabilities- Probability due to data computation and probability due to offered bandwidth.

\section{Conclusion}

Mobile cloud computing is one of the mobile technologies that combine the advantages of both mobile computing and cloud computing- providing optimal services for mobile users. This technology offers us much more efficient computing by centralizing data storage, processing and bandwidth. Since mobile systems have limited resources, hereby data offloading in cloud may alleviate these limitations. It is important to consider path delay along with queue delay while taking offloading decision. It is obvious that if we follow the algorithm stated in this paper, it will both save our time and energy; and increase the performance of mobile computation technology.

\section{References}

[1] M. Armbrust, A. Fox, R. Griffith, A. D. Joseph, R. Katz, A. Konwinski, and M. Zaharia, "A view of cloud computing," Communications of the ACM, vol. 53, pp. 50-58, 2010. View at Google Scholar | View at Publisher

[2] V. Namboodiri, "Towards sustainability in portable computing through cloud computing and cognitive radios," presented at the 39th International Conference on Parallel Processing Workshops, 2010.

[3] V. Cardellini, V. D. Personé, V. D. Valerio, F. Facchinei, V. Grassi, F. L. Presti, and V. Piccialli, "A game-theoretic approach to computation offloading in mobile cloud computing," Mathematical Programming, vol. 157, pp. 421-449, 2015.

[4] A. M. Hatami, M. Mirmohseni, and F. Ashtiani, "A new data offloading algorithm by considering interactive preferences," presented at the 2016 IEEE 27th Annual International Symposium on Personal, Indoor, and Mobile Radio Communications (PIMRC), 2016. 
[5] Y. Kwon, H. Yi, D. Kwon, S. Yang, Y. Cho, and Y. Paek, "Precise execution offloading for applications with dynamic behavior in mobile cloud computing," Pervasive and Mobile Computing, vol. 27, pp. 58-74, 2016. View at Google Scholar $\mid$ View at Publisher

[6] X. Liu, C. Yuan, Y. Li, Z. Yang, and B. Cao, "A lightweight algorithm for collaborative task execution in mobile cloud computing," Wireless Personal Communications, vol. 86, pp. 579-599, 2015. View at Google Scholar $\mid$ View at Publisher

[7] K. Kumar, J. Liu, Y. Lu, and B. Bhargava, "A survey of computation offloading for mobile systems," Mobile Networks and Applications, vol. 18, pp. 129-140, 2012. View at Google Scholar $\mid$ View at Publisher

[8] F. Xia, F. Ding, J. Li, X. Kong, L. T. Yang, and J. Ma, "Phone2cloud: Exploiting computation offloading for energy saving on smartphones in mobile cloud computing," Information Systems Frontiers, vol. 16, pp. 95-111, 2013. View at Google Scholar | View at

[9] R. K. Balan, Simplifying cyber foraging. Pittsburgh, PA: School of Computer Science, Carnegie Mellon U, 2006.

[10] R. Roostaei and Z. Movahedi, "Mobility and context-aware offloading in mobile cloud computing," presented at the 2016 Intl IEEE Conferences on Ubiquitous Intelligence \& Computing, Advanced and Trusted Computing, Scalable Computing and Communications, Cloud and Big Data Computing, Internet of People, and Smart World Congres (UIC/ATC/ScalCom/CBDCom/IoP/SmartWorld), 2016.

[11] X. Chen, "Decentralizes computation offloading game for mobile cloud computing," IEEE Transactions on Parallel and Distributed Systems, vol. 26, pp. 974-983, 2015. View at Google Scholar $\mid$ View at Publisher 